\title{
Automation Technique for the Plunger in Liquid Cell of X-Band Microwave Bench Using Stepper Motor
}

\author{
S. S. Nalwad ${ }^{1}$, S. V. Vedpathak ${ }^{2}$, Dr. K. U. Gore ${ }^{2}$ \\ ${ }^{1}$ Department of Electronics, N.E.S. Science College, Nanded.(Maharashtra)India \\ ${ }^{2}$ Head Department of Physics, N.S.B.College, Nanded. .(Maharashtra)India
}

\begin{abstract}
Automation is the use of Computer controlled stepper motors. It is one of the most versatile forms of positioning systems. Generally dielectric parameters of different polar liquids, binary mixture of polar liquid with non polar liquid in different mole fraction or weight fraction are measured by using X-band Microwave bench manually. The measurements done manually are not much accurate and exact. Hence, personal computer (PC)based automatic measurement technique is used for accurate measurement of the dielectric parameters. PC based stepper motor moves the plunger in liquid cell of microwave bench \& measures the distance up to fraction of mm to nanometer per step. This technique is used to give the maximum accuracy for the measurement of $\lambda d$. Further accuracy of this technique can be increased when the stepper motor is operated in micro stepping mode.
\end{abstract}

Keywords :- Driver, Microcontroller, PC, Stepper motor, X-band Microwave bench

\section{Introduction:}

The operations done by human beings are replaced with automatic control system which consists of the machines, advanced electronic techniques, and computers . Manually the plunger in liquid cell of X-band microwave bench can move approximately $1 \mathrm{~mm}$ at a time in forward or backward direction, Stepper motors are widely used in applications requiring precise motions using computer control. Special logic and high-current drive circuits are required to drive stepper motors, PC is used to control the steps of the stepper motor in clockwise and anticlockwise direction. It is also used to display the movement of stepper motor clockwise and anticlockwise direction.

This application describes the use of single microcontroller to control the speed, direction and rotation angle of a stepper motor by sending pulse sequences to the motor winding in response to the control commands. Commands executed by the code in this application include: single step motor rotates in a clockwise or counter clockwise direction ,The sequence of input pulses is directly related to the direction of motor shaft rotation. The speed of motor shaft rotation is directly related to frequency of the input pulses and the length of rotation .It is directly related to the number of input pulses applied. The PC based automation technique gives the maximum accuracy.

\section{Stepper Motor :}

Stepper motor is one of the most useful devices in the automation . It forms the most convenient and versatile bridge between a set of motion rules . It can be made to move slowly, quickly, in reverse, pause, complete revolutions, partial revolutions and even individual steps of less than a degree of rotation. With this flexibility of movement coupled with an abundance of torque for relatively little power applied.

A Stepper Motor which divides a full rotation into a number of steps. when fixed DC voltage is applied to it, a step motor rotates in discrete step angles. The Stepper Motors are manufactured with micro steps per revolution and Gear reducers may be used to increase resolution of positioning and micro steps from 400 to 25600 per revolution can be used. The motor's position can be commanded to move and hold at one of these steps without any feedback sensor.

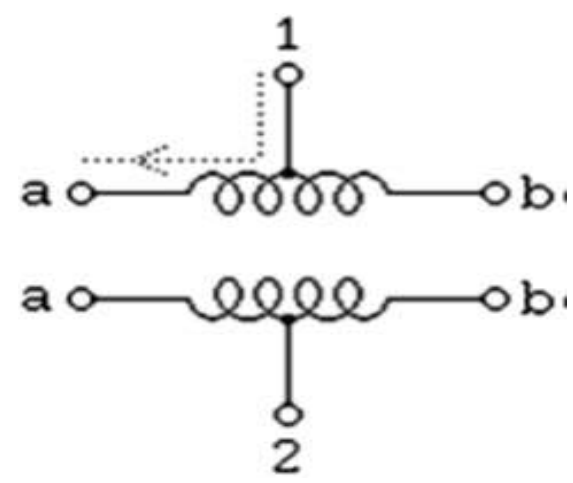

Fig.1: Unipolar Stepper Motor Coil

* Part of this work was presented in the national conference on "Nanotechnology-2012 held at M.. M,Nilanga (Maharashtra),07-08 Sept.2012. Also another part of this work is accepted in ISCA 2013, kolkata 
Automation Technique for the plunger in Liquid cell of X-band Microwave bench Using Stepper

The stepper motor used here, has 5 leads , 4 leads representing the four stator windings and $5^{\text {th }}$ terminal is used for centered-tapped lead. As the sequence of power is applied to each stator winding the rotor will rotate. There are several widely used sequences where each has a different degree of precision. In the full step sequence, two coils are energized at the same time and motor shaft rotates. The coils are energized in order to rotate stepper motor in clockwise or anticlockwise direction .As the micro steps become smaller, motor operation becomes smooth, thereby greatly reducing resonance in any parts.

The system is built around the microcontroller. In this system a unipolar stepper motor is used with following. Unipolar stepping motors with 5 or 6 wires are available and usually wired as shown in Fig. 1 with a center tap at terminal 1 and 2 on each of the two windings. In use, the center taps of the windings are typically wired to the positive supply and the two ends of each winding are alternately grounded to reverse the direction of the field provided by that winding.

\section{Experimental Details : Functional Block Diagram:}

Automation technique consists of, personal computer , logic circuit , microcontroller ,motor driver , stepper motor and X-band microwave bench with liquid cell ,but automation technique moves the plunger in liquid cell by fraction of $\mathrm{mm}$ or in nanometer per step. When the stepper motor is operated in stepping mode in forward or backward direction as per digital pulses given to it., gives the maximum accuracy. Further accuracy of this technique can be increased when the stepper motor is operated in micro stepping mode.

The PC is used to operate the stepper motor and by software program written in $\mathrm{C}++$ language , the stepper motor displaces the plunger in liquid cell of the microwave bench in proper direction The distance covered by the plunger in liquid cell of the microwave bench forward or backward direction is also displayed on the screen of monitor. Specifications: Maximum voltage: 24V DC, 1Amp and Step angle: 1.8 degree Steps per revolution. The block diagram of PC based measurement technique is shown in Fig. 2.

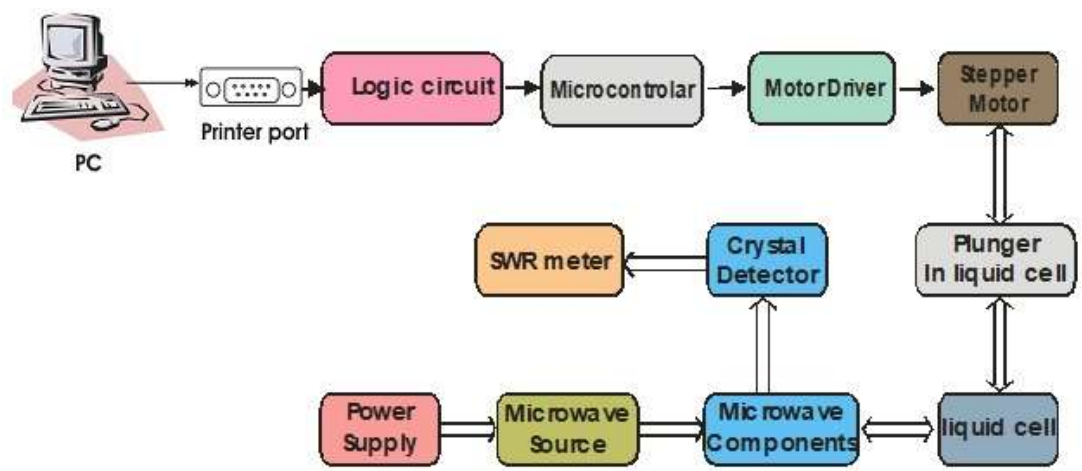

Fig.2: Functional Block Diagram

\section{Parallel Port :}

The stepper motor is interfaced with personal computer through the parallel printer port .The ports are identified in personal computer by their base address, which are nearly fixed for all the class of IBM clones. The addresses are $378 \mathrm{H}, 278 \mathrm{H}$ and others. Generally a personal computer has only one printer port with port address $378 \mathrm{H}$ - the port used in this work for the control of stepper motor's rotation . The data bits (D0-D7) can be accessed at the base address, i.e., $378 \mathrm{H}$.The data to be given out by the personal computer is output at this base address, also called as the base register. To rotate the stepper motor in clockwise direction, we have to provide two values to the port address $378 \mathrm{H}$, the values are 2 and 0 respectively, but in these two values we have to provide some time delay. To rotate the stepper motor in anticlockwise direction, we have to provide two values to the port address $378 \mathrm{H}$, the values are 3 and 1 respectively, but in these two values we have to provide some time delay.

\section{Mechanical set-up of Liquid cell :}

The high reliability and high accuracy rolled ball screw (4) of length $300 \mathrm{~mm}$ with ball nut(5). The ball screw eliminates clearance between the ball nut and screw which avoids back-lash error, and reduces elastic deformation. The ball screw will get much better rigidity with accuracy. The rolled ball screw has lead of $4 \mathrm{~mm}$. The ball screw is fixed vertically between two ball screw support units(3) in the Mechanical assembly (6) with leveling screws shown in fig1. The axel of stepper motor is coupled with the rolled ball screw with the help of coupler unit (2). The plunger (8) in Liquid cell (9) of Microwave bench (10), is coupled(7) with the ball nut. The Stepper motor (1) is. connected to driver and control circuit. 

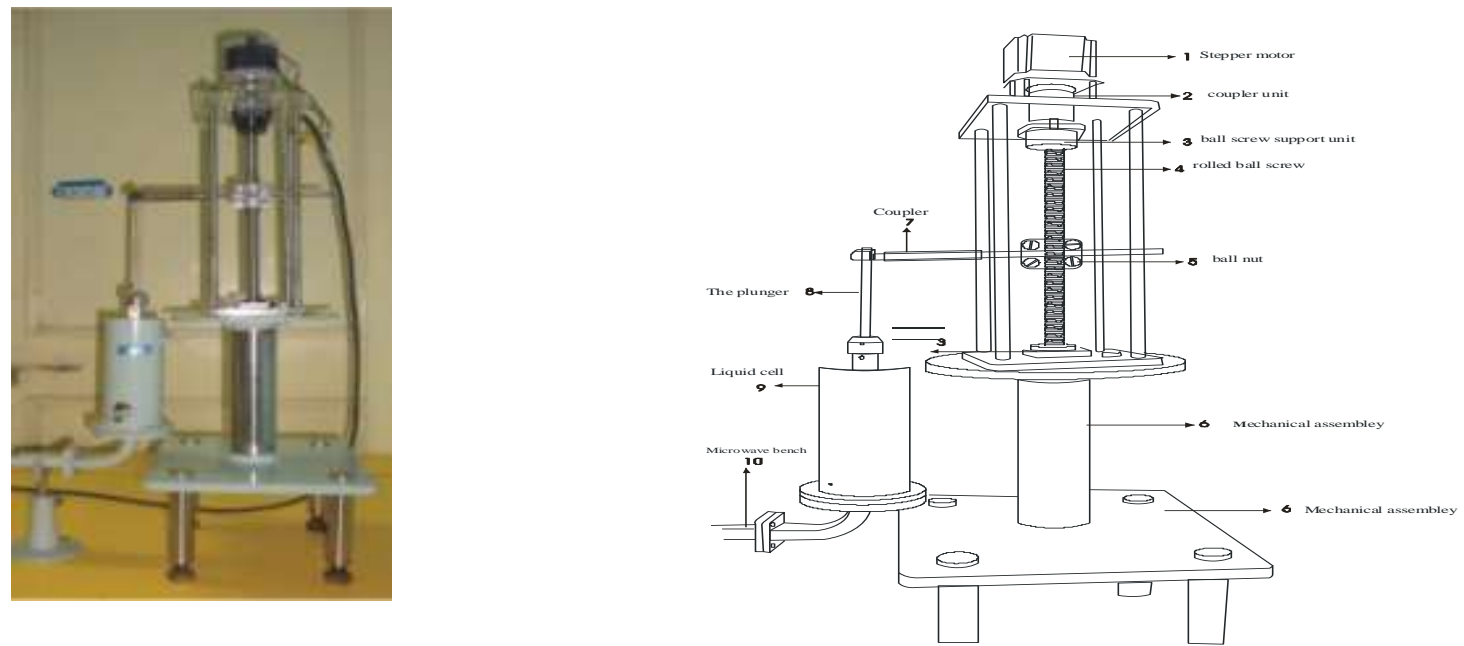

Fig.3: Experimental set-up with Labeling for Liquid cell of X-band Microwave bench

\section{Observations:}

The measurements of different physical parameters are measured by using Microwave bench Hence personal computer (PC) based automatic measurement technique is used for accurate measurement of positions. The clockwise or anticlockwise rotation of the motor are controlled through PC. The position (starting or ending) are measured as shown in Table 1, using the PC based automatic measurement technique. PC based stepper motor moves the plunger in liquid cell of microwave bench as shown in fig. 3 .

Table-1: Observation table for readings of Lengths in mm by using micro steps 25600 per revolution of stepper motor

\begin{tabular}{|l|l|l|l|l|l|l|l|l|l|}
\hline R .No & Length & R.No & Length & R .No & Length & R .No & Length & R .No & Length \\
\hline 1 & 0.000156 & 16 & 0.0025 & 31 & 0.004844 & 46 & 0.007188 & 61 & 0.009531 \\
\hline 2 & 0.000313 & 17 & 0.002656 & 32 & 0.005 & 47 & 0.007344 & 62 & 0.009688 \\
\hline 3 & 0.000469 & 18 & 0.002813 & 33 & 0.005156 & 48 & 0.0075 & 63 & 0.009844 \\
\hline 4 & 0.000625 & 19 & 0.002969 & 34 & 0.005313 & 49 & 0.007656 & 64 & 0.01 \\
\hline 5 & 0.000781 & 20 & 0.003125 & 35 & 0.005469 & 50 & 0.007813 & 65 & 0.010156 \\
\hline 6 & 0.000938 & 21 & 0.003281 & 36 & 0.005625 & 51 & 0.007969 & 66 & 0.010313 \\
\hline 7 & 0.001094 & 22 & 0.003438 & 37 & 0.005781 & 52 & 0.008125 & 67 & 0.010469 \\
\hline 8 & 0.00125 & 23 & 0.003594 & 38 & 0.005938 & 53 & 0.008281 & 68 & 0.010625 \\
\hline 9 & 0.001406 & 24 & 0.00375 & 39 & 0.006094 & 54 & 0.008438 & 69 & 0.010781 \\
\hline 10 & 0.001563 & 25 & 0.003906 & 40 & 0.00625 & 55 & 0.008594 & 70 & 0.010938 \\
\hline 11 & 0.001719 & 26 & 0.004063 & 41 & 0.006406 & 56 & 0.00875 & 71 & 0.011094 \\
\hline 12 & 0.001875 & 27 & 0.004219 & 42 & 0.006563 & 57 & 0.008906 & 72 & 0.01125 \\
\hline 13 & 0.002031 & 28 & 0.004375 & 43 & 0.006719 & 58 & 0.009063 & 73 & 0.011406 \\
\hline 14 & 0.002188 & 29 & 0.004531 & 44 & 0.006875 & 59 & 0.009219 & 74 & 0.011563 \\
\hline 15 & 0.002344 & 30 & 0.004688 & 45 & 0.007031 & 60 & 0.009375 & 75 & 0.011719 \\
\hline
\end{tabular}

\section{Software :}

The software program is written in different programming language but we use $\mathbf{C + +}$ language for microcontroller, because this language work like low, middle and high level language .Also in this language we can make executable files and It works as per flow chart shown in Fig. 3. The rotation of motor is controlled through printer port of computer and is received by microcontroller. The microcontroller loads step sequence to rotate stepper motor in clockwise or anticlockwise direction. The RPM of the motor can also be increased or decreased by changing the time delay 


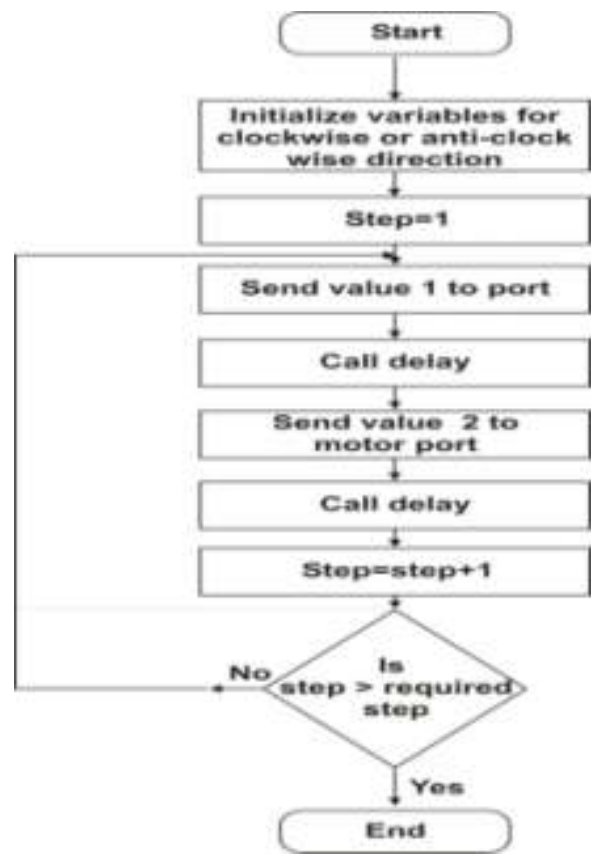

Fig. 4: Flow chart for $\mathrm{C}++$ program

\section{Conclusion}

The accuracy of the measurement of dielectric parameters such as permittivity, permittivity loss, Loss tangent , Thermodynamic parameters etc. of solids or Organic Liquids by using X-band Microwave bench can be increased to high extent. This measurement depends on only the measurement of guide wavelength $\lambda d$.The use of micro stepping for a standard stepper motor is presented in this paper. The key feature of this controller lies in its flexibility to provide for high position resolution operation of a given stepper motor control system. In this case their existing standard stepper motor controller to a high position resolution controller, For the measurement of $\lambda d$ in this method analog to digital Converter (ADC) may be used for data acquisition in PC. The same stepper motor may be used to the probe in slotted section of the microwave bench.

\section{Journal Papers:}

\section{References:}

[1] Mandeep Singh, Rekha \& Balwinder Singh International Journal of computer science \& communication Vol. 1, No. 1, JanuaryJune 2010, pp. 189-191

[2] R.H. Amenerkar, C.S. Adgaonkar, S.S. Yawale and S.P. Yawale, "Microwave properties of vanadium borate glasses", Material Science., 25, No. 5, October 2002, pp 431-434.

\section{Books:}

[3] E. Balguruswamy: Programming in ANSI C, TMH, New Delhi, 1999

[4] Y.P. Kanetkar: Let Us C, BPB Publications, New Delhi, 1999.

[5] M. Kulkarni, Microwave and Radar Engineering, Third Edition, Umesh Publication, 2004-2005, pp 274276.

[6] Von Hippel“Dielectric materials and applications", John and Sons New York, 1954, pp 104-115..

[7] Duglas V .Hall. "Microprocessor Interfacing and Programming”.McGraw-Hill.1998

[8] Mazidi Muhammad Ali,“The 8051 Microcontroller and Embedded Systems”, Prentice Hall of India, New Delhi, India, pp. 492507, Chap 17, 2007.

[9] B. Hedges: Industrial Robotics, Jaico publishing House (1993)..

[10] B. Govindarajalu: IBM PC and Clones: Hardware, Troubleshooting and Maintenance,TMH, New Delhi, Ch. 3, pp. 63-102 (1998).

\section{Proceedings Papers:}

[11] Baluta, G. Coteata, M., "Precision Micro stepping System for Bipolar Stepper Motor Control", IEEE International Conference on Electrical Machines and Power Electronics,pp. 291 - 296, Sept. 2007.

[12] Rusu, C. Szoke, E, "Embedded System Design for Field-Oriented Stepper Motor Control", IEEE International Conference on Optimization of Electrical and Electronic Equipment, pp.63 - 68, May 2008.

[13] Rusu, C. Birou, I. Szoke, E, "Model Based Design Controller for the Stepper Motor", IEEE International Conference on Automation, Quality and Testing, Robotics,2, pp.175 - 179, May 2008. 